\title{
An Active View Planning Method for Mobile Robots Using A Trinocular Visual Sensor
}

\author{
Min Y. Kim ${ }^{\mathrm{a}}$ and Hyungsuck Cho ${ }^{\mathrm{a}}$ \\ ${ }^{\mathrm{a}}$ Department of Mechanical Engineering, Korea Advanced Institute of Science and Technology
}

\begin{abstract}
The ability of mobile robots to perceive and recognize environments is essential for autonomous navigation. To improve the performance of autonomous environment perception for mobile robots, it is important to effectively plan the next pose (position and orientation) of the sensor system at a current navigation state. In this paper, we propose a nextview-planning method for autonomous map construction needed for mobile robots with visual range sensor systems. The proposed view-planning method mimics the decision-making method of human beings, and uses the occlusion information reduced from the geometric relationship between the sensor view and objects as an important clue for the next sensor view planning. The proposed view-planning algorithms are developed in the following steps: 1) Given a prior map and range measurements sensed at a current location of the mobile robot, it is determined which parts in the map are interested in a view of solving the map uncertainty. 2) Based on the selected potential regions, some candidate poses of the sensor system for the next environment sensing are carefully generated. 3) The created candidates are evaluated by using a specially designed evaluation parameter, and the best one of them is selected as a next sensor position based on a fuzzy decision-making method. In this work, the principle of the view planning method is described in detail, and a series of experimental tests is performed to show the feasibility of the method for autonomous map building. For sensing the environments, an active trinocular vision sensor using laser structured light is utilized, which is mounted on the pan-tilt mechanism of the mobile robot, which is composed of a laser stripe projector and two cameras.
\end{abstract}

Keywords: Autonomous map building, Sensor view planning, Active trinocular vision, Mobile robot navigation

\section{INTRODUCTION}

For the autonomous navigation of mobile robots in unknown space, two major functions established on mobile robots are self-localization and map-building. These two procedures are closely related, and each procedure affects the other. The self-localization is a procedure that a wheel based-service robot called "mobile robot" estimates its pose at a current state, and the map building that the robot builds its own information on the environment. Actually, a variety of sensors have been used for the self-localization and the map-building. Recently, the vision sensor and laser range finder are widely used due to their high measurement accuracy and robustness. Generally, the localization is executed by matching the features extracted from the current measurements with a given map information. This technique is called feature-based localization using the given map of the navigation environment, and the map information is indispensable for this localization. The previous studies for the localization have been concentrated on the case that an a prior map is given on the navigation environment [1][2]. Recently, the simultaneous localization and map building methods are researched, which does not need a prior map [3][4]. But, these are not for the navigation purpose of mobile robots in unknown space, such as the autonomous map building or the short path finding to the target position with the acquisition of the environment information in unknown space.

Generally, for building a complete map in unknown space, it is necessary to obtain observation views of the object from various positions and orientations, so that all data describing the map is acquired. Since the view angle and range of most

*mykim@1ca.kaist.ac.kr; phone +82-42-869-3253; fax +82-42-869-3210; Dept. Mech. Eng., Korea Advanced Institute of

Science and Technology, 373-1, Guseung-dong, Yuseung-gu, Daejon, Korea 
sensor systems are limited and range data acquisition is a time-consuming process, a method of automating process obtaining the range data would be desirable. When we use a vision sensor system, occlusion problem frequently occurs by geometric and optical reason. This makes it further difficult to automate the range data obtaining process. In this situation, the next-view-planning is a process of selecting the next view position and orientation of a sensor system. In computer vision society [5]-[12], various view-planning algorithms have been proposed for dealing with the object modeling or inspection problem. The methods for view planning can be divided in generate-and-test approach and synthesis approach [8]. In generate-and-test approach [5]-[9], the potential next view candidate points are generated and evaluated to decide which view is the best with respect to the task constraints. In synthesis approach [10], [11], the task requirements are characterized as the types of the inequality constraints, and the sensor location values that satisfy the task constraints are directly determined from these analytical relationships. In this paper, we propose a view planning method following generate-and-test paradigm.

In this paper, we begin by giving a brief explanation on our sensor systems, and then describe our method to determine next view position of the sensor systems for the autonomous map building of mobile robots. Finally, a series of experiments for 2D-map building using the proposed algorithm are performed, and the experimental results are discussed in views of the effectiveness and usefulness of this method in detail.

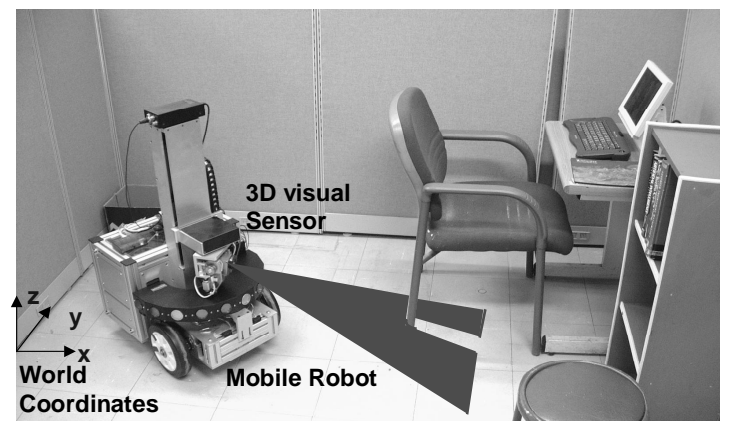

(a) concept of the map building process

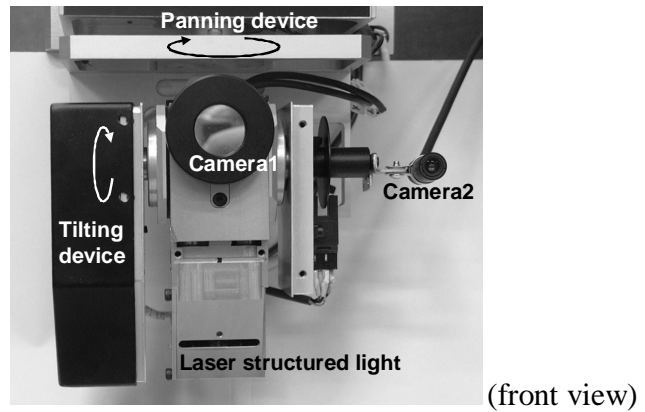

(b) active trinocular sensor system for environment perception

Fig. 1 Autonomous navigation for mobile robots

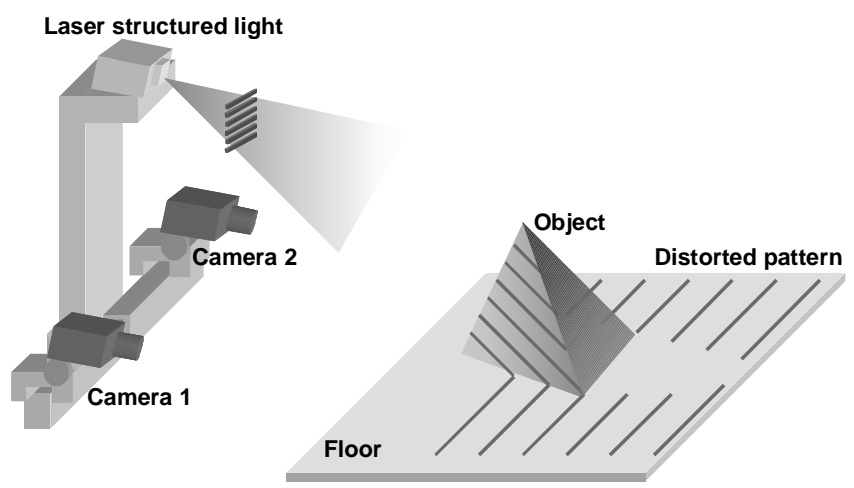

(a) schematic diagram of the sensor system

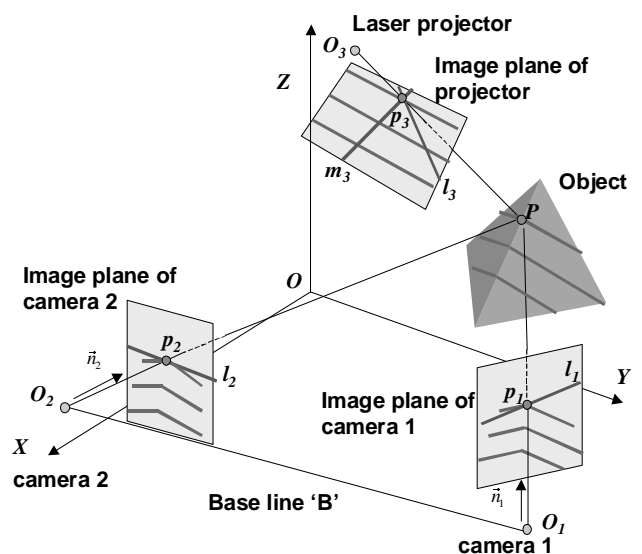

(b) correspondence matching by using three epipolar constraints

Fig. 2 Principle of the range measure for the active trinocular vision sensor

\section{AUTONOMOUS MAP-BUILDING}

\subsection{Mobile robot and sensor system}

The mobile robot used in this paper is shown in Fig. 1. For autonomous navigation in unknown space, it is equipped with 
a number of sensors, e.g., ultrasonic sensors, infrared sensors, and an active trinocular vision sensor. During the navigation process, it perceives the navigation environment by using these sensors. For obtaining the detail range information on environment, the active trinocular vision sensor is utilized [13]. It is based on the optical triangulation principle and the trinocular vision theory, which is composed of two CCD cameras, and a structured laser light as shown in Fig. 1 (b). The laser structured light is projected on a scene of interest, and then two cameras composing a stereo vision system take the pattern image of the scene onto which the laser is projected. By modeling the laser structured light module as another camera and using the epipolar constraints that these three cameras constitute, the correspondences between the line features in two input images can be established without difficulty. In addition to it, since the laser is used for the active illumination, the sensor system provides the robust measurement with variations of nature of the environment and the easy extraction of the interested feature information for sensing and modeling objects. Fig. 2 shows a schematic diagram of the sensor system and the basic principle of the range measure for this sensor. Imagine a point $P$ on the object. $O X Y Z$ denotes the world coordinates system. $O_{1}, O_{2}$, and $O_{3}$ represent the optical centers of two cameras and the projector. Assume that the object point $P$ on which the pattern is projected is observable from two cameras simultaneously. When a 3D point $P$ of the object is observed as the image point $p_{1}$ on the image of camera 1 , the matching point $p_{2}$ on the image of camera 2 must lie on the epipolar line $l_{2}$ that is the projection of the line $O_{1} p_{1}$ onto the image plane of camera 2. As shown in Fig. 2 (b), the epipolar line $m_{3}$ is a constraint that the relation of the projector and camera 2 make on virtual image plane for image point $p_{2}$, and the epipolar line $l_{3}$ is the other constraint that the relation of the projector and camera 1 make on the same plane for image point $p_{1}$. If the image point $p_{1}$ and the image point $p_{2}$ are a pair of corresponding points, these epipolar lines must intersect at a point $p_{3}$ that is on the line feature of the virtual multi-stripe laser image. Using this characteristic, correspondence candidate points can be checked one by one. When the object point $\mathrm{P}$ on which the pattern is projected is visible at two cameras, these constraints are always effective. After correspondence matching, 3D coordinates of an object point $P$ are easily computed using stereo vision theory. This laser vision sensor has some range error with variations of object distance. For example, when the distance between the mobile robot and the object is $0.7 \mathrm{~m}, 1.4 \mathrm{~m}$, and $2.1 \mathrm{~m}$, the error standard deviations are $4.1 \mathrm{~mm}, 7.4 \mathrm{~mm}$, and $12.8 \mathrm{~mm}$, respectively. This error is mainly due to the image processing error and the quantization effect of the imaging sensor, and tends to increase with the object distance.

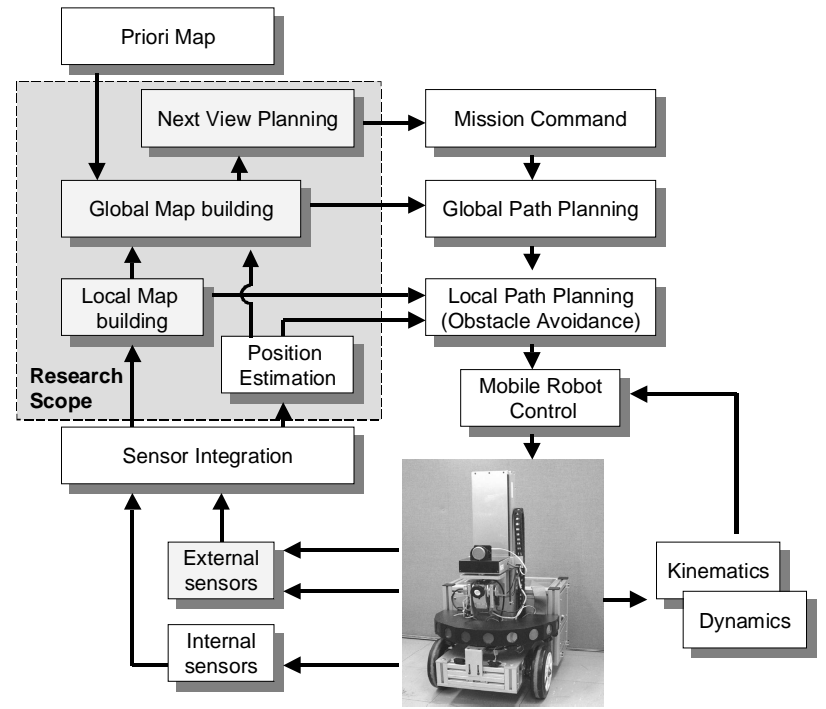

Fig. 3 A functional block diagram for autonomous navigation of mobile robots

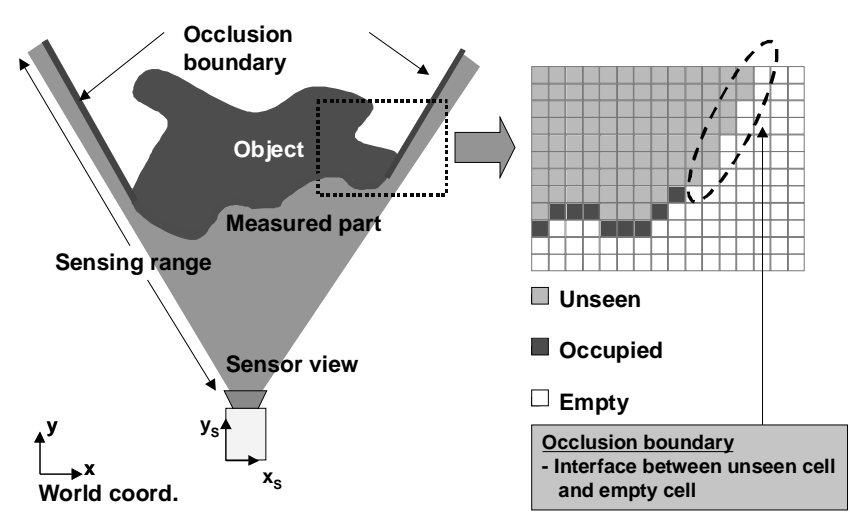

Fig. 4 Object sensing and state mapping on grid map

\subsection{Navigation and view planning}

Fig. 3 shows a functional block diagram for autonomous navigation of mobile robots, and the research scope of this paper is blocked. The navigation flow of mobile robots in unknown space is as follows: 1) Given an a prior map and a global map, the next-view-planning process generates the mission command for the autonomous navigation of mobile 
robots. 2) By analyzing the current global map, the robot plans a safe path trajectory from the current robot position to the next goal position. 3) During the navigation, the robot moves along this path with avoiding unpredictable obstacles. 4) At a new robot position, it perceives newly the environment using various sensors, and a local map is constructed in a robot coordinate frame. 5) The localization process is performed with matching the local map to the global map, and then the current global map is updated. Through this navigation framework, the robot explores the environment and builds the map concurrently by patching together the local models acquired by the range sensor into the global map.

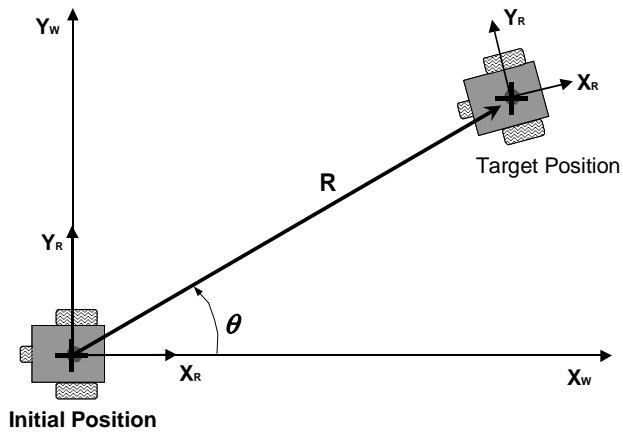

(a) selection of an initial and target position

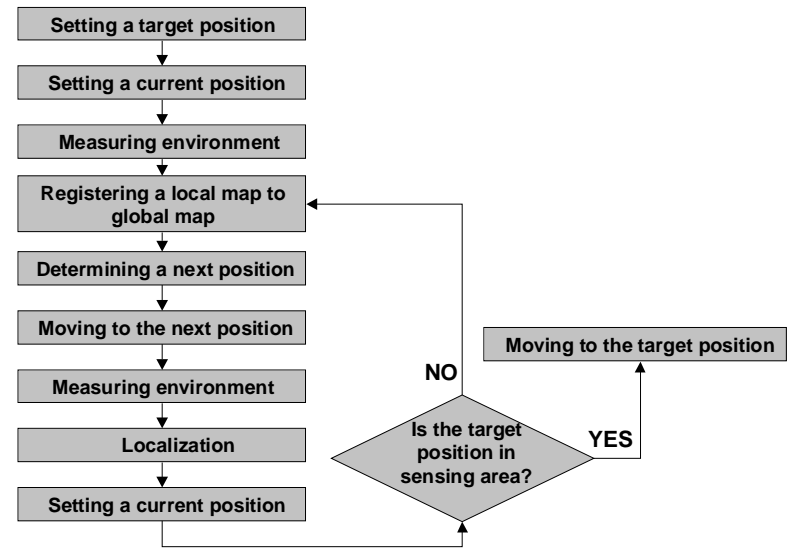

(b) algorithmic flowchart for sequential map building and localization

Fig. 5 Autonomous map building during navigation

\section{A VIEW PLANNING METHOD USING AN ARTIFICIAL INTELLIGENCE METHOD}

\subsection{Map representation and Navigation strategies}

In this paper, it is assumed that the navigation environment for the mobile robot is mainly composed of polygonal objects that are frequently observed in the indoor environment, and we assume that the object shapes are equivalent in the orthogonal direction to the floor. Therefore, we are able to project them on two-dimensional navigation map, and to make the mobile robot navigation restrict on this map for simplicity. The proposed navigation method consists of autonomous map building module and self-localization module. Former is the algorithm to decide a next sensor viewpoint for the successful navigation at current state, and latter is to decide where the robot is. For efficient operation of two modules, we utilize a hybrid map of consisting of the line map and the grid map. The used self-localization method [14] is categorized into the feature-based localization. Geometric features easily extracted from such polygonal environments are the corner feature formed by two planes and the line feature of each plane of polygonal objects. We utilize these features for self-localization of mobile robots. However, a presentation form of the navigation map is selected as a grid map for the next-view planning module. Different from the approach of Gonzalez and Latombe [15], the reason using the grid map is that the high computational cost is unnecessary to represent and find occlusion and object areas. The occlusion area results from the sensing limit of the sensor system and the invisible area due to the relation between objects and the viewing direction of sensor. It plays an important role to decide the sensor viewpoint for the autonomous map building. For the rapid and effective map building, the sensor must be located in a place where can eliminate the occlusion boundary greatly. An example for sensing an object and mapping its states on the grid map is shown in Fig. 4. In the grid map, the occlusion boundary is defined as the interface line between the unseen area and the empty one as shown in the figure. Since the map of the navigation environment is not given before navigation, the mobile robot should build gradually an environment map during navigation, and uses it for self-localization. Therefore, we adopt an autonomous map building method that is based on the next-view-planning algorithm proposed in [16]. The navigation map is built under an additional constraint that it is necessary to observe more than two features at a next robot position for the localization as described in [14]. If less than two features are measured, the robot localization fails on the previous map, so it is impossible to add a local map to the global map. The navigation purpose of the mobile robot is that 
it executes autonomously the map building and localization on the exploration range of interest between the initial position and the target position, which is determined by the range $R$ and direction $\theta$ in Fig. 5 (a). When the mobile robot executes the sequential map building and localization, the assigned tasks are shown in Fig. 5 (b).

\subsection{View Candidate Generation}

The proposed next-view planning algorithms to decide a next viewpoint of the robot follows generate and test paradigm, and the whole framework is represented in Fig. 6. This method consists of four steps: 1) making candidate points for next position and orientation of the sensor system using the current map information, 2) checking these points whether the mobile robot with sensor system can be placed or not, 3) evaluating the remaining candidate points on the welldefined cost function, and 4) selecting the best one as the next point of placing the sensor system. Here, we select a fuzzy decision maker for evaluating and selecting the candidates. Based on the sensory information and the map information, the fuzzy decision maker selects the optimal view point, which best satisfies multiple map-building goals, among a set of view candidates. Because fuzzy decision making can generate the optimal compromise between even conflicting objectives, this approach can guide the robot to achieve often conflictive, two map-building goals: map expanding and target point reaching. The concept of this approach is similar to selecting the view-point for next observation in human exploration. In this work, the generation method of the robot localization candidates and the occlusion rejection candidates is not described due to space limitation. The detail description on the candidate generation can be found in references [14][16].

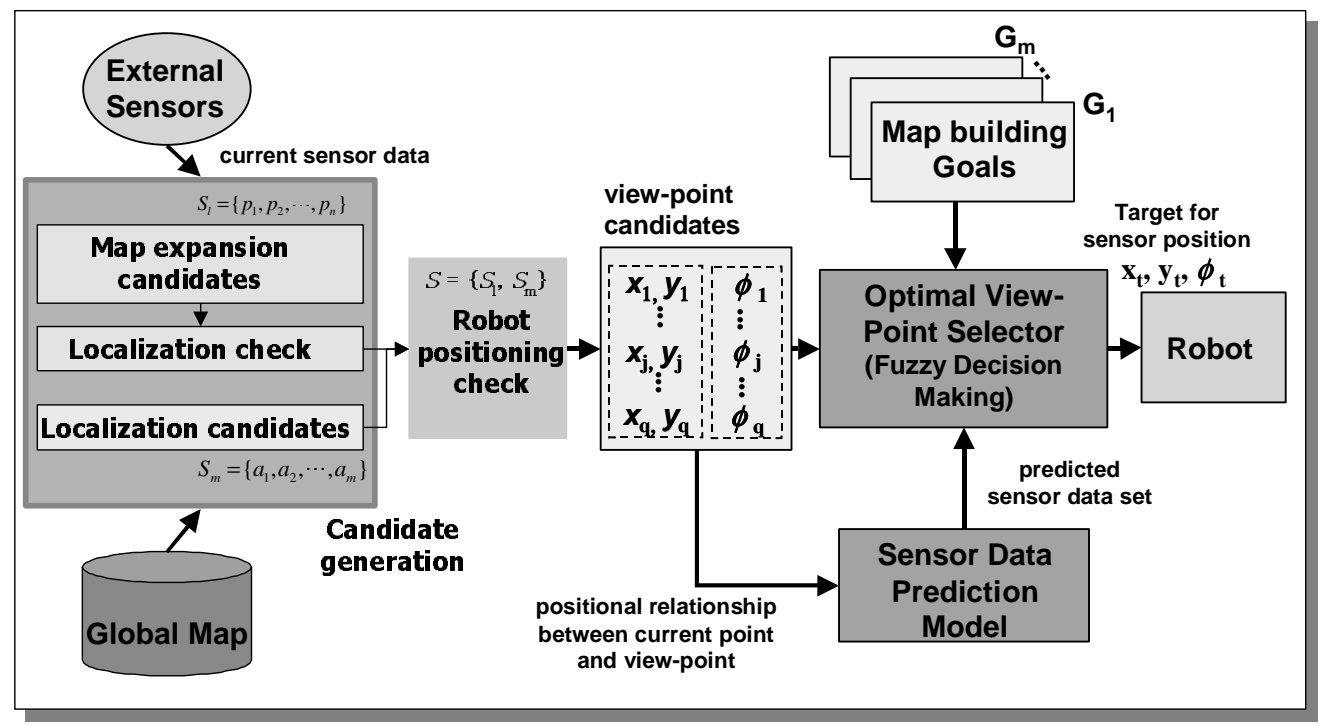

Fig. 6 Algorithmic flow of next-view-planning process

The pose (position and orientation) of the candidate is represented through three-dimensional vector $p=(x, y, \phi)$, where $x$ and $y$ are Cartesian coordinates with respect to world coordinate system, and $\phi$ is the sensor orientation. The generated view candidates for localization, which is described in the reference [14], are as follows:

$$
p_{i}=\left\{x_{i}, y_{i}, \phi_{i}\right\} \quad \text { for } i=\{1, \cdots, n\} \quad, \quad S_{l}=\left\{p_{1}, p_{2}, \cdots, p_{n}\right\}
$$

where $n$ denotes the total number of the view candidates for localization. Secondly, the map expansion candidates are generated through the method described in the reference [16]. Basically, these are determined by occlusion boundary information on the current map. Its distribution on the map, the connected line segments of it, and the nearest occlusion point are considered for solving the occlusion problem. Total number of the candidates depends on the current map situation. The generated map expansion candidates are as follows: 


$$
a_{i}=\left\{x_{j}, y_{j}, \phi_{j}\right\} \quad \text { for } j=\{1, \cdots, m\}, \quad S_{m}=\left\{a_{1}, a_{2}, \cdots, a_{m}\right\}
$$

where $m$ denotes the number of the view candidates for the map expansion. Thirdly, all of the candidates, $S=\left\{S_{\mathrm{l}}, S_{\mathrm{m}}\right\}$, are checked whether the mobile robot with sensor system can be placed at each of them or not, because our research is that of determining next position of the mobile robots with the sensor position. If this positioning constraint is satisfied, the candidate point is confirmed for the evaluation process. Otherwise, some changes to it are allowed. If any pose variation of the robot does not satisfy it, this candidate is removed from the candidate point set. Then, generated candidates are evaluated by two evaluation criterions, and the next position is decided as a candidate with the highest score based on a fuzzy decision making method. The evaluation criteria are composed of two components: 1) Visibility criterion aims at maximizing the amount of occlusion cell solved by next view, and 2) Distance criterion aims at minimizing the distance of the mobile robot motion.
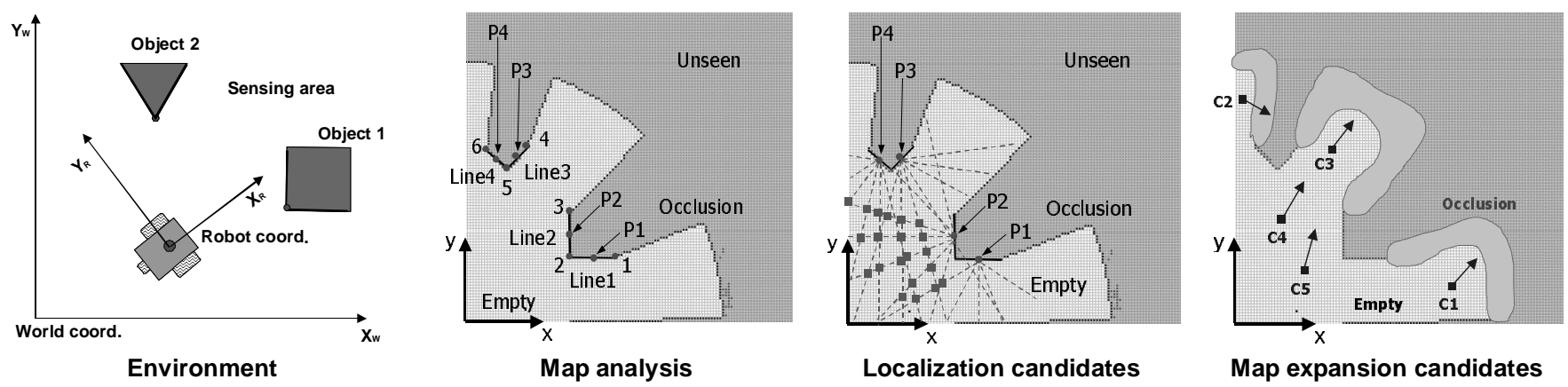

Fig. 7 An example for the generation of view candidates

\subsection{Decision-making technique for the selection of the best candidate}

In the proposed method, for the evaluation of the generated candidates, two criteria are defined. The first criterion, called the visibility criterion, aims at maximizing the amount of occlusion cell seen by next view. The other criterion, called distance criterion, aims at minimizing the distance from a view point to the navigation target point. Bellman and Zadeh [17] considered a mathematical model for the decision-making and suggested a fuzzy decision model that is often used by human being. They considered a decision making process in which the goals and/or the constraints are fuzzy in nature. According to their formulation, objective functions (or goals) and constraints can be characterized by their membership functions. The decision in a fuzzy environment can be considered as the intersection of fuzzy objective (or goals) functions and fuzzy constraints. Fig. 8 describes the pictorial concept of fuzzy decision-making.

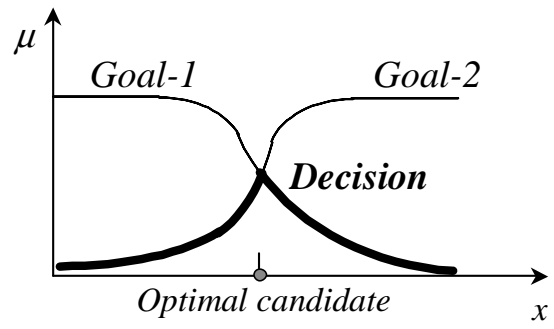

Fig. 8 Pictorial concept of fuzzy decision making

\section{1) Decision-making theory}

Multi Attribute Decision Making (MADM) deals with the decision making in a discrete decision space [18]. Let $X=\left\{x_{1}, \ldots, x_{n}\right\}$ be the set of decision alternatives, and $\tilde{G}_{j}$ for $j=1, \ldots, m$, be the fuzzy sets of representing fuzzy goals. When the attainment of the goal $\tilde{G}_{j}$ by alternative $x_{\mathrm{i}}$ can be expressed by the degree of membership $\mu_{\tilde{G}_{j}}\left(x_{i}\right)$, the decision set $\tilde{D}$ can be defined as the intersection of all fuzzy goals as follows: 


$$
\tilde{D}=\tilde{G}_{1} \cap \tilde{G}_{2} \cap \cdots \cap \tilde{G}_{m}
$$

Then, we select the $x_{\mathrm{i}}$ with the largest degree of membership in $\tilde{D}$ as the optimal alternative.

Saaty [19] proposed a hierarchical procedure for determining the weights of the fuzzy goals by computing eigenvectors of the matrix $\mathbf{M}$ of relative weights of subjective estimates. The relative importance, $\alpha_{\mathrm{i}}$, of the goal among themselves is established by pairwise comparison, and is arranged in a matrix $\mathbf{M}$.

$$
\mathbf{M}=\left[\begin{array}{cccc}
\frac{\alpha_{1}}{\alpha_{1}} & \frac{\alpha_{1}}{\alpha_{2}} & \cdots & \frac{\alpha_{1}}{\alpha_{m}} \\
\frac{\alpha_{2}}{\alpha_{1}} & \frac{\alpha_{2}}{\alpha_{2}} & \ldots & \frac{\alpha_{2}}{\alpha_{m}} \\
\vdots & \vdots & & \vdots \\
\frac{\alpha_{m}}{\alpha_{1}} & \frac{\alpha_{m}}{\alpha_{2}} & \cdots & \frac{\alpha_{m}}{\alpha_{m}}
\end{array}\right]
$$

By employing Saaty's eigenvector method to matrix $\mathbf{M}$, consistent weights $w_{j}$ for each goal can be determined. The degrees of goal attainment, $\mu_{\hat{G}_{j}}\left(x_{i}\right)$, are weighed exponentially by the respective $w_{j}$. Thus, the resulting fuzzy sets are $\left(\tilde{G}_{j}\left(x_{i}\right)\right)^{w_{j}}$. Finally we determine the intersection of all $\left(\tilde{G}_{j}\left(x_{i}\right)\right)^{w_{j}}$ as follows:

$$
\widetilde{D}=\widetilde{G}_{1}^{w_{1}} \cap \widetilde{G}_{2}^{w_{2}} \cap \cdots \cap \widetilde{G}_{m}^{w_{m}},
$$

and select the $x_{i}$ with largest degree of membership in $\tilde{D}$ as the optimal alternative.

\section{2) Fuzzy goal for autonomous map building}

The autonomous map building goals for a mobile robot can be summarized as (i) map expanding and (ii) fast reaching to target point by using map information. These goals can be expressed linguistically as (i) to place to acquire enough map information, and (ii) to proceed toward the target point using map information. The goal function is designed with considering two factors:

- $A_{k}:$ amount of the uncertain cells eliminated at the $k^{\text {th }}$ candidate point

- $D_{k}:$ distance to the target position from the $k^{\text {th }}$ candidate point.

The amount of the eliminated occlusion can be defined as follows:

$$
A_{k}=\text { total number of elements of set } U \cap V_{k} \quad \text { for } k=\{1, \cdots, n+m\}
$$

where $U$ is the union of the set of occlusion boundary cells and the set of unseen cells, and $V_{k}$ is the set of the cells predicted to be visible from the $k^{\text {th }}$ candidate point.

Using the current map information and predicted map information at next view pose, we can define three fuzzy goals for the autonomous map building as follows:

$$
\begin{array}{ll}
\mathrm{G}_{1}: & A_{k} / A_{\max }>C_{1} \\
\mathrm{G}_{2}: & D_{\min } / D_{k}>C_{2}
\end{array}
$$

where $C_{1}$ and $C_{2}$ are constants, which contain the designer's intention for the decision making process. $A_{\max }$, and $D_{\min }$ are the maximum of the occlusion elimination, and the minimum target distance among candidates, respectively. Fuzzy goal $G_{1}$ is defined for map expanding and $G_{2}$ for target point reaching.

Using exponential sigmoid functions, which is smoothly differentiable, membership functions of goals $G_{1}$ and $G_{2}$ have been designed as follows (see Fig. 9): 


$$
\mu_{\hat{G}_{1}}\left(x_{i}\right)=\frac{1}{1+\exp \left[-d_{1} \cdot\left(A_{k} / A_{\max }-C_{1}\right)\right]} \quad \text { and } \quad \mu_{\tilde{G}_{2}}\left(x_{i}\right)=\frac{1}{1+\exp \left[-d_{2} \cdot\left(D_{\min } / D_{k}-C_{2}\right)\right]}
$$

where $d_{1}$, and $d_{2}$ are appropriate constant scale factors that affect the sensitivity of the membership functions.
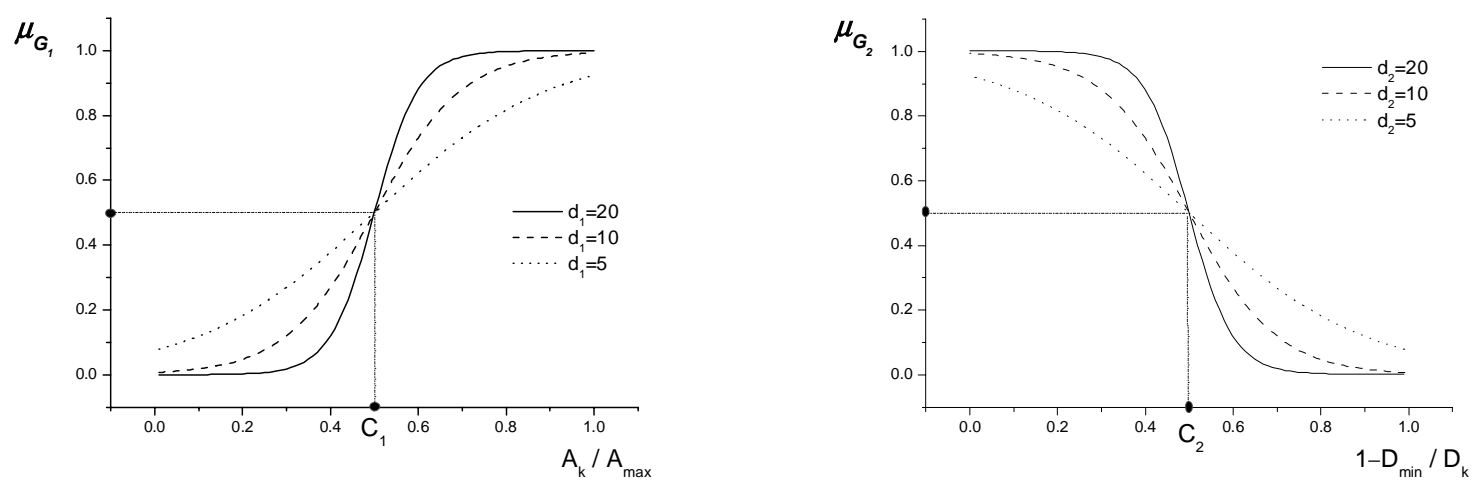

Fig. 9 Membership functions for two fuzzy goals

3) Selection of best view point

Now we can select the optimal via-point since we have goal attainments of each via-point candidate using predicted sensor data and robot-to-target distance. From Eq. (5), the membership of the decision set can be expressed using minmax operation as follows:

$$
\mu_{\tilde{D}}=\mu_{\tilde{G}_{1}{ }^{w_{1}}} \wedge \mu_{\tilde{G}_{2}{ }^{w_{2}}} \wedge \cdots \wedge \mu_{\tilde{G}_{m}{ }^{w_{m}}}
$$

Among the via-point candidates, one with the highest value of $\mu_{\tilde{D}}$ is selected as the optimal alternative.

\section{EXPERIMENTS FOR AUTONOMOUS MAP-BUILDING}

For the experiment, we used an environment composed of some polygonal objects and walls. The navigation environment is shown in Fig. 10 (a). Through experiments, we examine the relationship between the weights in the equation (5), the map building, and robot navigation. In Fig. 10 (b) and (c), it is shown how changes of the parameters $w_{1}$, and $w_{2}$ in the fuzzy decision making give effects on the map building and robot navigation behavior. If the weight for the eliminated occlusion is larger as shown in second figure, the mobile robot tends to move to a place at which more occlusion is eliminated at each decision time. Therefore, the purpose of the navigation is focused on the map building for information expansion in an unknown environment. Although the navigation path becomes complex with increment of $w_{l}$, the information on the environment becomes abundant. If the weight for the distance to the target position is larger as shown in third figure, the mobile robot tends to select the nearest candidate to the target position among the generated candidates. In this case, we obtain a navigation effect that the mobile robot moves along a short path to the target position and acquires the map information on that path. Therefore, we notice that two parameters of $w_{1}$ and $w_{2}$ function as critical factors in the proposed navigation method. In the figures, S and E indicate the initial and target position, respectively, and $\mathrm{N}_{j}$ denotes the $j$ th viewing position and direction. 


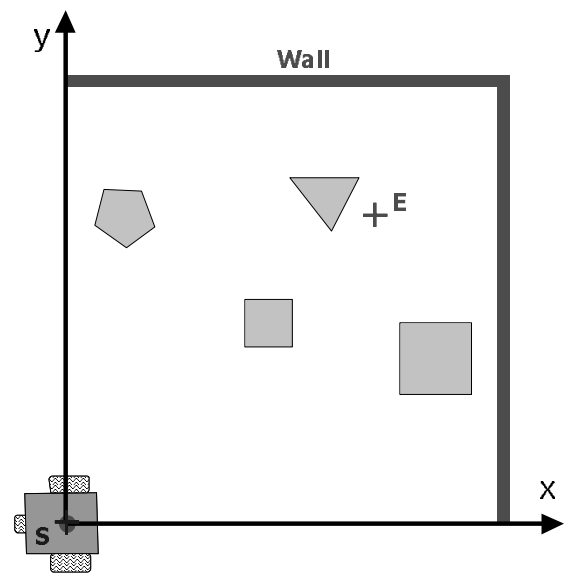

a) An environment for the experiments

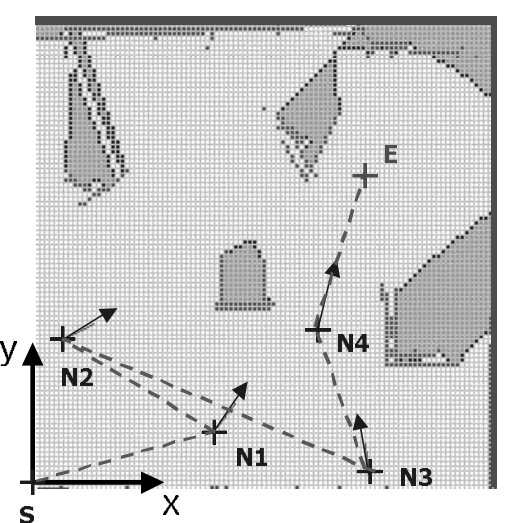

b) larger weight for first goal

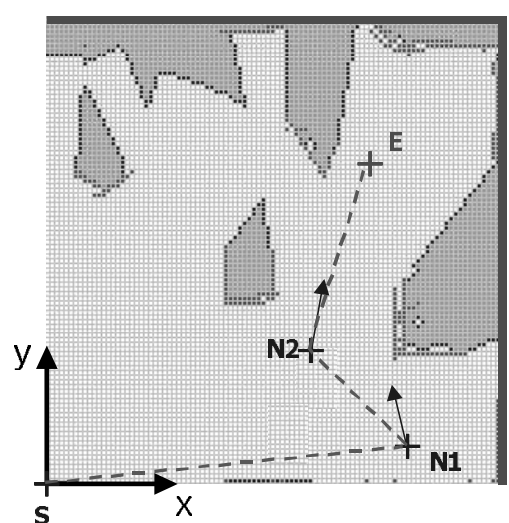

c) larger weight for second goal

Fig. 10 Effect on the map building and robot navigation with variations of relative importance between $w_{1}$ and $w_{1}$

\section{CONCLUSION}

A major research issue associated with mobile service robots is the creation of autonomous environment sensing and recognition system for navigation and task execution that is robust and efficient. We proposed an autonomous robot navigation method based on the sequential map building and localization with view planning function in unknown space. This algorithm assumes that the navigation environment is mainly composed of a number of polygons. To estimate the posture of the mobile robot, it utilizes the features (corners and lines) of the polygonal environment. At a robot position, the acquired feature information is matched with the current map information. The matching is based on interpretationtree search method. Specially, the next-view-planning algorithm was proposed for autonomous map building during the navigation to target point. Using these methods, some candidates were generated for the sequential map building and localization, and the next position of the mobile robot was determined by evaluating through the proposed decisionmaking method. The fuzzy goals for autonomous map building was designed, based on two navigation criteria. If the weight for the occlusion elimination is larger, the mobile robot tends to build a map of the navigation environment completely. If the weight for the distance to the target position is larger, the mobile robot tends to move along a short path to the target position and build the map on that path only. The performed experiments show the successful navigation of the mobile robot with the proposed navigation method.

\section{REFERENCES}

[1] A. Escalera, L. Moreno, M. A. Salichs and J. M. Armingol, "Continuous mobile robot localization by using structured light and geometric map,” International Journal of Systems Science, Vol.27, No.8, pp.771 782, 1996.

[2] G. K. Shaffer, A. Stentz, W. L. Whittaker and K. W. Fitzpatrick, "Position Estimator for Underground Mind Equipment," IEEE Trans. on Industry Applications, Vol.28, No.5, pp.1131 1140, 1992.

[3] A. J. Davison and D. W. Murry, "Simultaneous Localization and Map-Building Using Active Vision," IEEE Transactions on Pattern Analysis and Machine Intelligence, Vol.24, No.7, July, 2002.

[4] E. Zalama, G. Candela, J. Gómez and S. Thrun, "Concurrent Mapping And Localization For Mobile Robots With Segmented Local Maps", Proceeding of IEEE/RSJ International Conference on Intelligent Robots and Systems EPFL, Lausanne, Switzerland, pp.546 551, 2002.

[5] C. I. Connolly, "The determination of next best views," Proc. IEEE Int. Conf. on Robotics and Automation, pp. 432-435, 1985. 
[6] J. Maver and R. Bajcsy, "Occlusion as a guide for planning the next view," IEEE Trans. on Pattern Analysis and Machine Intelligence, 15(5), pp. 417-433, May 1995

[7] R. Pito, "A sensor-based solution to the "next best view" problem," Proceedings of International Conference on Pattern Recognition, pp.941-945, 1996

[8] M. K. Reed, P. K. Allen, and I. Stamos, "Automated model acquisition from range images with view planning," Proceedings of International Conference on Computer Vision and Pattern Recognition, pp. 72-77, Puerto Rico, June 1997.

[9] H. Zha, K. Morooka, T. Hasegawa, and T. Nagata, "Active modeling of 3-d objects: Planning on the next best pose for acquiring range images," Proceedings of International Conference on Recent Advances in 3-D Digital Imaging and Modeling, pp. 68-75, Ottawa, Canada, May 1997.

[10] C. K. Cowan and P. D. Kovesi, “Automatic Sensor Placement from Vision Task Requirements,” IEEE Trans. Pattern Analysis and Machine Intelligence, Vol. 10, pp. 407-416, May 1988.

[11] R.Y. Tsai and K. Tarabanis, "Occlusion-free Sensor Placement Planning," in Machine Vision for Three-Dimensional Scenes, H. Freeman, Academic, 1990.

[12] K.A. Tarabanis, P.K. Allen, and R.Y. Tsai, "A survey of sensor planning in computer vision” IEEE Trans. on Robotics and Automation, 11(1), pp. 86-104, Feb. 1995.

[13] M.Y. Kim, H.S. Cho, and J.H. Kim, "3D Sensor system using multi-stripe laser and stereo camera for environment recognition of mobile robots,” Proceedings of SPIE, Optomechatronic Systems III, Vol. 4902, pp. 542-552, 2002.

[14] M.K. Kim, M.Y. Kim, and H.S. Cho, "A Mobile Robot Navigation Method Based on Sequential Autonomous Map Building and Self-Localization Using a Laser Vision Sensor," Proceedings of $8^{\text {th }}$ International Conference on Rehabilitation Robotics, pp. 9699, 2003.

[15] H.H. Gonzales-Banos, and J. Latombe, "Navigation Strategies for Exploring Indoor Environments," International Journal of Robotics Research, Vol. 21, No. 10-11, pp. 829-848, 2002.

[16] O.K. Lee, M.Y. Kim, H.S. Cho, and K. C. Koh, "Next View Planning of autonomous map building for mobile robots," Proceedings of 2002 FIRA Robot World Congress, pp. 273-277, 2002.

[17] R.E. Bellman and L.A. Zadeh, "Decision-making in a fuzzy environment," Management Science, Vol.17, No.4, pp.B-141 - B164, 1970.

[18] H.J. Zimmermann, Fuzzy set theory and its applications 2nd ed., Kluwer Adademic Publishers, 1991.

[19] T.L. Saaty, "Exploring the Interface Between Hierarchies, Multiple Objectives and Fuzzy Sets," Fuzzy Sets and Systems, Vol. 1, pp. 57-68, 1978. 